\title{
Enhanced production of 2,3-butanediol by engineered Saccharomyces cerevisiae through fine-tuning of pyruvate decarboxylase and NADH oxidase activities
}

\author{
Jin-Woo Kim', Jungyeon Kim², Seung-Oh Seo ${ }^{3}$, Kyoung Heon Kim² ${ }^{2}$ Yong-Su Jin ${ }^{3}$ and Jin-Ho Seo ${ }^{*}$
}

\begin{abstract}
Background: 2,3-Butanediol (2,3-BD) is a promising compound for various applications in chemical, cosmetic, and agricultural industries. Pyruvate decarboxylase (Pdc)-deficient Saccharomyces cerevisiae is an attractive host strain for producing 2,3-BD because a large amount of pyruvate could be shunted to 2,3-BD production instead of ethanol synthesis. However, 2,3-BD yield, productivity, and titer by engineered yeast were inferior to native bacterial producers because of the following metabolic limitations. First, the Pdc-deficient yeast showed growth defect due to a shortage of $\mathrm{C}_{2}$-compounds. Second, redox imbalance during the 2,3-BD production led to glycerol formation that lowered the yield.

Results: To overcome these problems, the expression levels of Pdc from a Crabtree-negative yeast were optimized in S. cerevisiae. Specifically, Candida tropicalis PDC1 (CtPDC1) was used to minimize the production of ethanol but maximize cell growth and 2,3-BD productivity. As a result, productivity of the BD5_G1CtPDC1 strain expressing an optimal level of Pdc was 2.3 folds higher than that of the control strain in flask cultivation. Through a fed-batch fermentation, $121.8 \mathrm{~g} / \mathrm{L}$ 2,3-BD was produced in $80 \mathrm{~h}$. NADH oxidase from Lactococcus lactis (noxE) was additionally expressed in the engineered yeast with an optimal activity of Pdc. The fed-batch fermentation with the optimized 2-stage aeration control led to production of $154.3 \mathrm{~g} / \mathrm{L} 2,3-\mathrm{BD}$ in $78 \mathrm{~h}$. The overall yield of 2,3-BD was $0.404 \mathrm{~g} 2,3-\mathrm{BD} / \mathrm{g}$ glucose which corresponds to $80.7 \%$ of theoretical yield.
\end{abstract}

Conclusions: A massive metabolic shift in the engineered S. cerevisiae (BD5_G1CtPDC1_nox) expressing NADH oxidase was observed, suggesting that redox imbalance was a major bottleneck for efficient production of 2,3-BD by engineered yeast. Maximum 2,3-BD titer in this study was close to the highest among the reported microbial production studies. The results demonstrate that resolving both $C_{2}$-compound limitation and redox imbalance is critical to increase 2,3-BD production in the Pdc-deficient S. cerevisiae. Our strategy to express fine-tuned PDC and noxE could be applicable not only to 2,3-BD production, but also other chemical production systems using Pdc-deficient S. cerevisiae.

Keywords: Pyruvate decarboxylase, Saccharomyces cerevisiae, 2, 3-Butanediol, NADH oxidase, Metabolomics, Metabolic engineering

\section{Background}

2,3-Butanediol (2,3-BD) is a potential feedstock for use by the chemical, cosmetic, and agricultural industries.

\footnotetext{
*Correspondence: jhseo94@snu.ac.kr

1 Department of Agricultural Biotechnology, Center for Food and Bioconvergence, Seoul National University, Seoul 151-921, Republic of Korea

Full list of author information is available at the end of the article
}

It is used as a precursor for synthesizing 1,3-butadiene which is a monomer for producing synthetic rubber. In addition, 2,3-BD could be used as anti-freeze agent (R,R-BD), solvents, and its derivatives could be used as flavoring agent, moisturizer, liquid fuel additive, humectant in cosmetics, and plant growth promoter $[1,2]$. 
Many research groups have attempted producing 2,3$B D$ via microbial fermentations with native bacterial producers such as Klebsiella pneumoniae, K. oxytoca, Paenibacillus polymyxa, and Enterobacter species [3, 4]. These bacterial strains are able to produce 2,3-BD with high productivity, but formation of biofilm consisting of exopolysaccharides [5], optical impurity of 2,3-BD [3], and production of various by-products such as succinate, lactate, acetate, and ethanol [6] hampered the use of the strains for industrial fermentations. Commercialization is also constrained by most of 2,3-BD-producing bacteria belonging to class II (pathogenic) microorganisms, which requires tight safety regulations for industrial-scale fermentations [7]. In contrast, Saccharomyces cerevisiae is a GRAS (generally recognized as safe) microorganism and has been widely employed in industrial-scale fermentation processes for producing various chemicals and fuels. Thus, S. cerevisiae would be an appropriate microorganism for industrial production of 2,3-BD. Nonetheless, it is necessary to delete the genes coding for pyruvate decarboxylase (Pdc) for 2,3-BD production because $S$. cerevisiae produces ethanol as a major product.

Pdc-deficient $S$. cerevisiae is a promising metabolic background for producing non-ethanol products such as 2,3-BD, 3-hydroxypropionic acid, and lactic acid. It accumulates pyruvate which is a precursor of numerous chemical molecules instead of producing ethanol from glucose [8]. However, impaired growth of Pdc-deficient $S$. cerevisiae on glucose has been a major obstacle to exploit Pdc-deficient $S$. cerevisiae for 2,3-BD production. The reasons for the growth defect are (1) lack of acetyl-CoA in the cytosol $[9,10]$ and (2) a redox imbalance due to accumulation of cytosolic NADH [11, 12]. Cytosolic acetyl-CoA is indispensable for growth of $S$. cerevisiae because it is used for synthesizing lysine and fatty acids in the cytosol [8-10]. Pdc-deficient $S$. cerevisiae cannot synthesize cytosolic acetyl-CoA from glucose because the deletion of PDC leads to elimination of cytosolic $\mathrm{C}_{2}$-compounds (e.g., acetaldehyde, acetate, ethanol). Within mitochondria, the pyruvate dehydrogenase (Pdh) complex converts pyruvate into acetyl-CoA, but mitochondrial acetyl-CoA cannot pass through the inner membrane of mitochondria [13]. Although the YBR219C and YBR220C are known as putative genes coding for acetyl-CoA transporter, activities of these enzymes were not sufficient for supplying enough acetyl-CoA to cytosol [14]. Therefore, cell growth and carbon utilization of Pdcdeficient $S$. cerevisiae strains were greatly inhibited by insufficient supply of cytosolic acetyl-CoA, which is often termed as ' $\mathrm{C}_{2}$-auxotrophy'. Redox imbalance is another reason for growth defect of Pdc-deficient S. cerevisiae on glucose. Excess NADH is generated in Pdc-deficient $S$. cerevisiae because oxidation of cytosolic NADH via the ethanol production pathway is blocked. NADH generated by converting glucose to pyruvate should be re-oxidized to $\mathrm{NAD}^{+}$to maintain cellular redox metabolism. However, insufficient activity of the respiratory pathway because of the glucose-induced Crabtree effect [11] and absence of transhydrogenase activity $[15,16]$ aggravate the redox imbalance of Pdc-deficient $S$. cerevisiae.

There have been several reports about the production of 2,3-BD with engineered $S$. cerevisiae harboring the bacterial 2,3-BD biosynthetic enzymes [12, 17, 18]. By introduction of $B$. subtilis $\alpha$-acetolactate synthase (als $S$ ) and $\alpha$-acetolactate decarboxylase $(a l s D)$, and overexpression of endogenous 2,3-butanediol dehydrogenase $(B D H 1)$, pyruvate was converted into optically pure (2R,3R)-BD by the Pdc-deficient S. cerevisiae with MTH1 mutation [12].

The mutation (G241C) in MTH1 has been reported to suppress the growth defect of the Pdc-deficient S. cerevisiae strain [12]. In the presence of extracellular glucose, signal transduction via the glucose sensors (Rgt2/Snf3) and casein kinases (Yck1/2) induces phosphorylation of Mth1 to be degraded [19]. The degradation of Mth1 led to the down-regulation of hexose transporter genes (HXTs) [20], resulting in decreased glucose influx rate in spite of extracellular glucose [21]. A slow glucose uptake rate caused by the $M T H 1$ mutation might be responsible for restoration of growth defect by the Pdc-deficient strains on glucose [12]. Additionally, although the exact mechanism remains unknown, the mutant MTH1 could partially relieve the $\mathrm{C}_{2}$-auxotrophy of Pdc-deficient $S$. cerevisiae [12, 21]. The mutation in MTH1 might be regarded as an indispensable strategy for Pdc-deficient $S$. cerevisiae to grow on glucose, but the slow glucose consumption rates caused by the MTH1 mutation resulted in much lower 2,3-BD productivity [12] than that by bacterial 2,3-BD production systems [22, 23]. The lower glucose consumption rates and 2,3-BD productivity by the Pdc-deficient $S$. cerevisiae are putatively caused by reduced expression levels of HXTs by MTH1 mutation $[19,20,24]$. As such, metabolic engineering strategies to alleviate the growth defect of Pdc-deficient S. cerevisiae without mutations in MTH1 needs to be devised for efficient and rapid production of 2,3-BD by engineered yeast.

In this study, we present metabolic engineering strategies for rapid production of 2,3-BD by the Pdc-deficient $S$. cerevisiae strain without mutating MTH1. To this end, we attempted to address both $\mathrm{C}_{2}$-auxotrophy and redox imbalance. For the construction of $\mathrm{C}_{2}$-independent strains, various $P D C$ genes from the Crabtree-negative yeast were expressed at a minimal level to eliminate the $\mathrm{C}_{2}$-auxotrophy of Pdc-deficient $S$. cerevisiae strains. As excess expression of $P D C$ produces a large amount of 
ethanol instead of producing 2,3-BD, we hypothesized that expression levels of $P D C$ need to be fine-tuned to balance metabolic fluxes between acetyl-CoA synthesis and 2,3-BD production. Specifically, $P D C$ expression levels were optimized through the variations of $P D C$ gene sources, promoters, and copy numbers. Additionally, Lactococcus lactis NADH oxidase (noxE) was coexpressed to resolve the issue of redox imbalance [18]. The resulting engineered $S$. cerevisiae strain expressing both PDC and noxE at optimal levels produced 2,3-BD with much higher titer, productivity, and yield than those of the other engineered yeast capable of producing 2,3$\mathrm{BD}$. The results presented in this study suggest that utilization of the engineered S. cerevisiae strain for 2,3-BD production could be an attractive alternative to bacterial 2,3-BD production systems [25].

\section{Results}

\section{A mutation in $\mathrm{MTH} 1$ decreased glucose uptake rate in Pdc-deficient S. cerevisiae}

The mutations in MTH1 were identified from evolved strains exhibiting improved growth phenotypes of Pdcdeficient S. cerevisiae. In particular, a mutation (G241C) was employed for improving 2,3-BD production by the $S$. cerevisiae strain because the MTH1 mutation could overcome the $\mathrm{C}_{2}$-auxotrophy of Pdc-deficient $S$. cerevisiae [12, 21]. However, the MTH1 mutation resulted in reduced expression levels of hexose transporters in S. cerevisiae [11, 20], causing slow glucose uptake rates. Batch fermentations were performed for comparing 2,3-BD production by 2,3-BD-producing strains with the $M T H 1$ mutation (the BD4 strain) and without the MTH1 mutation (the BD5 strain) (Additional file 1: Fig. S1). When glucose was used as a sole carbon source (Additional file 1: Fig. S1A, B), the maximum dry cell weight (DCW) of the BD4 strain was 5.5-fold higher $(2.2 \mathrm{~g} / \mathrm{L})$ than that of the BD5 strain $(0.4 \mathrm{~g} / \mathrm{L})$. The BD4 strain (with the MTH1 mutation) showed higher volumetric glucose uptake rate and $2,3-\mathrm{BD}$ productivity $(0.289 \mathrm{~g}$ glucose $/ \mathrm{L} \cdot \mathrm{h}$ and $0.087 \mathrm{~g}$ $2,3-\mathrm{BD} / \mathrm{L} \cdot \mathrm{h})$ than those $(0.155 \mathrm{~g}$ glucose $/ \mathrm{L} \cdot \mathrm{h}$ and $0.051 \mathrm{~g}$ 2,3-BD/L.h) of the BD5 strain (without the MTH1 mutation). However, when a small amount of ethanol $(0.5 \mathrm{~g} / \mathrm{L})$ was supplemented as a $\mathrm{C}_{2}$-compound (Additional file 1 : Fig. S1C, D), the maximum DCW of the both strains were similar ( 3.9 and $4.2 \mathrm{~g} / \mathrm{L}$ ), but the BD5 strain (without the MTH1 mutation) showed a much higher volumetric glucose uptake rate and 2,3-BD productivity (1.23 $\mathrm{g}$ glucose/L.h and $0.305 \mathrm{~g} 2,3-\mathrm{BD} / \mathrm{L} \cdot \mathrm{h}$ ) than the BD4 strain ( $0.665 \mathrm{~g}$ glucose/L.h and $0.205 \mathrm{~g}$ 2,3-BD/L.h). Thus, we concluded that the BD5 strain without the MTH1 mutation can grow and consume glucose with comparable to the wild type and produce 2,3-BD efficiently as long as $\mathrm{C}_{2}$-compounds are properly supplied.

\section{Optimization of $P D C$ expression levels led to enhanced} 2,3-BD production through relieving $C_{2}$-auxotrophy in Pdc-deficient S. cerevisiae

Partial restoration of Pdc might be a simple way to supply a $\mathrm{C}_{2}$-compound for relieving the $\mathrm{C}_{2}$-auxotrophy in Pdc-deficient S. cerevisiae. As shown in Additional file 1: Fig. S1, the addition of only a small amount of ethanol $(0.5 \mathrm{~g} / \mathrm{L})$ greatly enhanced $2,3-\mathrm{BD}$ productivity in the BD5 strain. This result suggests that fine-tuning of Pdc activity possibly maximizes $2,3-\mathrm{BD}$ productivity, while keeping the 2,3-BD yield unchanged by minimizing ethanol production. Therefore, a set of $P D C$ expression cassettes with different $P D C$ gene sources, promoters, and copy numbers was constructed to identify optimal levels of Pdc for enhanced 2,3-BD production without introducing MTH1 mutations. First, we searched for the Pdc enzymes with low activities in S. cerevisiae. As Crabtreenegative yeasts exhibit lower Pdc activities than Crabtreepositive yeasts [26-28], we reasoned that Pdc activities might be maintained at low levels even under excess glucose conditions when the $P D C$ genes from Crabtree-negative yeasts such as $C$. tropicalis and $K$. marxianus [26, 28] are optimally expressed. The kinetic parameters of $C$. tropicalis Pdc1 (CtPdc1), K. marxianus Pdc1, and endogenous Pdc1, Pdc5, and Pdc6 were examined after expressing each of the genes in the Pdc-deficient S. cerevisiae strain (Additional file 1: Table S1). As CtPdc1 showed the lowest $V_{\max }$ value among the five Pdc enzymes, the $C t P D C 1$ gene was selected to confer Pdc activity in the 2,3-BD-producing strain. The comparison of amino acid sequences of CtPdc1 and ScPdc1 was depicted in Additional file 1: Fig. S2. The Ctpdc1 and Scpdc1 proteins have $66 \%$ identical amino acid residues. Both Ctpdc1 and Scpdc1 proteins retain all structurally important amino acid residues for the $\mathrm{Mg}^{2+}$ and thiamine pyrophosphate (ThDP) binding loop (N473, N474, G476, D446, T392, S448, I479) and substrate activation (C223) that are conserved throughout all $P D C$ s [29-31]. In order to identify an optimal expression level of CtPDC1 for maximizing 2,3-BD production and minimizing ethanol production, the CtPDC1 gene under the control of various promoters in plasmids with different copy numbers was expressed in the BD5 strain (Table 1). Then, in vitro Pdc activities in the control and Pdc-expressed strains were measured (Additional file 1: Fig. S3). As expected, each strain exhibited different levels of Pdc activities. The Pdc activities of the BDt_C1CtPDC1, BD5_G1CtPDC1, BD5_C2CtPDC1, and BD5_T2CtPDC1 strains were 74.7, 122.1, 287.4, and $1387.0 \mathrm{mU} / \mathrm{mg}$ protein, respectively.

To evaluate the effects of different Pdc activities on 2,3-BD production by the engineered yeast, batch fermentations were carried out in minimal medium containing 90 g/L glucose (Fig. 1; Additional file 1: Fig. S4). 
Table 1 Strains and plasmids used in this study

\begin{tabular}{|c|c|c|}
\hline Strains and plasmids & Description & Reference \\
\hline \multicolumn{3}{|l|}{ Strains } \\
\hline Candida tropicalis ATCC20336 & Source for CtPDC1 & In this study \\
\hline Kluyveromyces marxianus KCTC17555 & Source for KmPDC1 & In this study \\
\hline Saccharomyces cerevisiae D452-2 & Source for SCPDC1, SCPDC5, SCPDC6 MATa leu2 his3 ura3 & [49] \\
\hline BD4 & D452-2, pdc1 1 , pdc5 4 , evolved 2,3-BD-producing strain & [12] \\
\hline SOS5 & $\mathrm{D} 452-2, p d c 1 \Delta, p d c 5 \Delta, p d c 6 \Delta$ & [18] \\
\hline SOS5_T2CtPDC1 & SOS5, p426TDH3_CtPDC1 & In this study \\
\hline SOS5_T2KmPDC1 & SOS5, p426TDH3_KmPDC1 & In this study \\
\hline SOS5_T2SCPDC1 & SOS5, p426TDH3_SCPDC1 & In this study \\
\hline SOS5_T2ScPDC5 & SOS5, p426TDH3_ScPDC5 & In this study \\
\hline SOS5_T2ScPDC6 & SOS5, p426TDH3_ScPDC6 & In this study \\
\hline BD5 & SOS5, p423_alsSalsD, p425_BDH1 & [18] \\
\hline BD5_Con & $\mathrm{BD} 5, \mathrm{p} 426 \mathrm{GPD}$ & [18] \\
\hline BD5_C1CtPDC1 & BD5, p406CYC1_CtPDC1 & In this study \\
\hline BD5_G1CtPDC1 & BD5, p406GPD2_CtPDC1 & In this study \\
\hline BD5_C2CtPDC1 & BD5, p426CYC1_CtPDC1 & In this study \\
\hline BD5_T2CtPDC1 & BD5, p426TDH3_CtPDC1 & In this study \\
\hline BD5_G1CtPDC1_nox & BD5, p406GPD2_CtPDC1, pAUR_LInox & In this study \\
\hline \multicolumn{3}{|l|}{ Plasmids } \\
\hline pRS406 & URA3 & [50] \\
\hline pRS426 & URA3 $2 \mu \mathrm{m}$ origin & [50] \\
\hline p406GPD2 & pRS406, GPD2 $2_{\text {prom }} C Y C 1_{\text {term }}$ & [18] \\
\hline p426CYC1 & pRS426, $C Y C 1_{\text {prom }} C Y C 1_{\text {term }}$ & [18] \\
\hline p426GPD & URA3 $2 \mu \mathrm{m}$ origin, $\mathrm{TDH}_{\text {prom }} \mathrm{CYC1} 1_{\text {term }}$ & [50] \\
\hline p423_alsSalsD & HIS3 $2 \mu \mathrm{m}$ origin, $T D H 3_{\text {prom }}$-alsS-CYC1 $1_{\text {term }} T D H 3_{\text {prom }}-a l s D-C Y C 1_{\text {term }}$ & [51] \\
\hline p425_BDH1 & LEU2 $2 \mu \mathrm{m}$ origin, $\mathrm{TDH}_{3}$ prom $^{-} \mathrm{BDH1}-\mathrm{CYC1}$ term & [12] \\
\hline p426TDH3_LInox & pRS426, TDH3 prom $-L /$ nox-CYC1 $1_{\text {term }}$ & [18] \\
\hline p426TDH3_CtPDC1 & pRS426, $T D H 3_{\text {prom }}-C t P D C 1-C Y C 1_{\text {term }}$ & In this study \\
\hline p426TDH3_KmPDC1 & pRS426, TDH3 prom $-K m P D C 1-C Y C 1_{\text {term }}$ & In this study \\
\hline p426TDH3_ScPDC1 & pRS426, TDH3 prom $-S C P D C 1-C Y C 1_{\text {term }}$ & In this study \\
\hline p426TDH3_ScPDC5 & pRS426, $T D H 3_{\text {prom }}-S C P D C 5-C Y C 1_{\text {term }}$ & In this study \\
\hline p426TDH3_ScPDC6 & pRS426, $T D H 3_{\text {prom }}-S C P D C 6-C Y C 1_{\text {term }}$ & In this study \\
\hline p406CYC1_CtPDC1 & pRS406, $C Y C 1_{\text {prom }}-C t P D C 1-C Y C 1_{\text {term }}$ & In this study \\
\hline p406GPD2_CtPDC1 & pRS406, GPD2 prom $-C t P D C 1-C Y C 1_{\text {term }}$ & In this study \\
\hline p426CYC1_CtPDC1 & pRS426, $C Y C 1_{\text {prom }}-C t P D C 1-C Y C 1_{\text {term }}$ & In this study \\
\hline PAUR_LInox & AUR1-C CEN6 ARS4, TDH3 prom $^{-L I n o x-C Y C 1} 1_{\text {term }}$ & In this study \\
\hline
\end{tabular}

The maximum DCW and glucose consumption rate increased after the expression of $C t P D C 1$. The maximum DCW $(1.04 \pm 0.06 \mathrm{~g} / \mathrm{L})$ of the BD5_G1CtPDC1 strain (CtPDC1 under GPD promoter and chromosomal integration) which has moderate Pdc activity among the engineered strains was 3.5-fold higher than the control strain $(0.30 \pm 0.00 \mathrm{~g} / \mathrm{L})$. While the BD5_G1CtPDC1 strain consumed $74.6 \pm 3.1 \mathrm{~g} / \mathrm{L}$ glucose in $120 \mathrm{~h}$ cultivation, the control strain consumed only $31.2 \pm 1.6 \mathrm{~g} / \mathrm{L}$ glucose during the same time period. The engineered strains showed different product profiles according to the $P D C$ expression levels. In contrast to the BD5_T2CtPDC1 (CtPDC1 under the TDH3 promoter in a multicopy plasmid) strain that produced a large amount of ethanol, the BD5_G1CtPDC1 strain produced less than $0.5 \mathrm{~g} / \mathrm{L}$ ethanol and exhibited the highest productivity among the engineered 2,3-BD-producing strains. The 2,3-BD productivity of the BD5_G1CtPDC1 strain $(0.18 \pm 0.01 \mathrm{~g} 2,3-\mathrm{BD} / \mathrm{L} \cdot \mathrm{h})$ was 2.3 -fold higher than that of the BD5_Con strain $(0.08 \pm 0.00 \mathrm{~g} 2,3-\mathrm{BD} / \mathrm{L} \cdot \mathrm{h})$. When fed-batch fermentation of the BD5_G1CtPDC1 strain was performed, $121.8 \mathrm{~g} / \mathrm{L}$ of $2,3-\mathrm{BD}$ was produced 


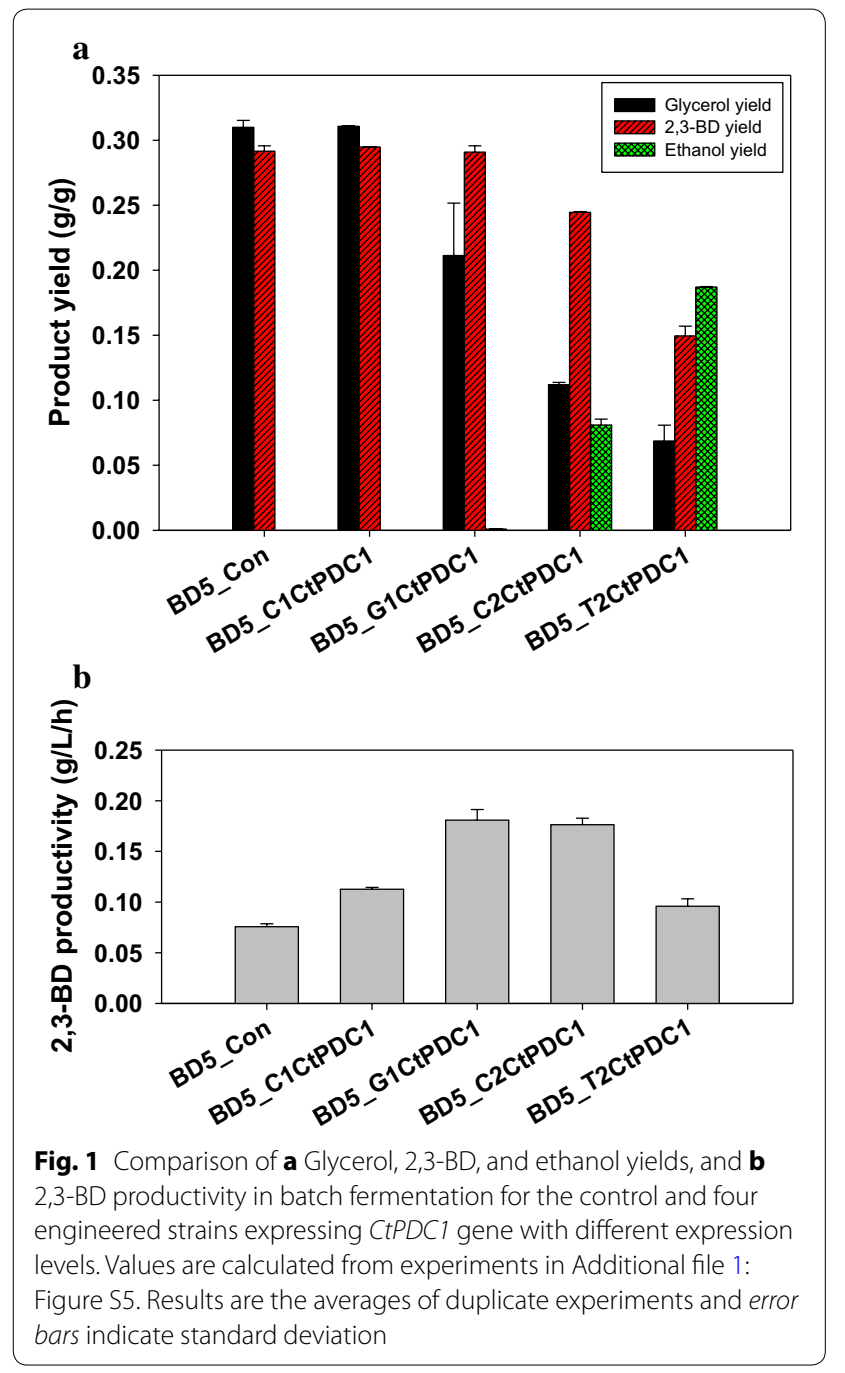

(Fig. 2). The volumetric productivity of 2,3-BD production reached $1.52 \mathrm{~g} 2,3-\mathrm{BD} / \mathrm{L} \cdot \mathrm{h}$ which is 3.9-fold higher than the case with the BD4 strain $(0.39 \mathrm{~g} 2,3-\mathrm{BD} / \mathrm{L} \cdot \mathrm{h})$ in a previous report [12]. Small amounts $(<1.0 \mathrm{~g} / \mathrm{L})$ of ethanol and acetoin accumulated, but a large amount of glycerol $(118.5 \mathrm{~g} / \mathrm{L})$ was produced as a by-product. A yield of glycerol $(0.333 \mathrm{~g}$ glycerol/g glucose $)$ was similar to that of 2,3-BD (0.337 g 2,3-BD/g glucose). These results suggest that optimal expression of $C t P D C 1$ can increase glucose uptake and 2,3-BD production rates substantially, but aggravate the yield of 2,3-BD production due to massive glycerol production.

\section{Expression of NADH oxidase increased 2,3-BD yield and productivity}

We reasoned that glycerol production during the 2,3$\mathrm{BD}$ production is due to surplus $\mathrm{NADH}$ production in the cytosol as the 2,3-BD-producing pathway is not redox-neutral [18]. Excess NADH in the cytosol should

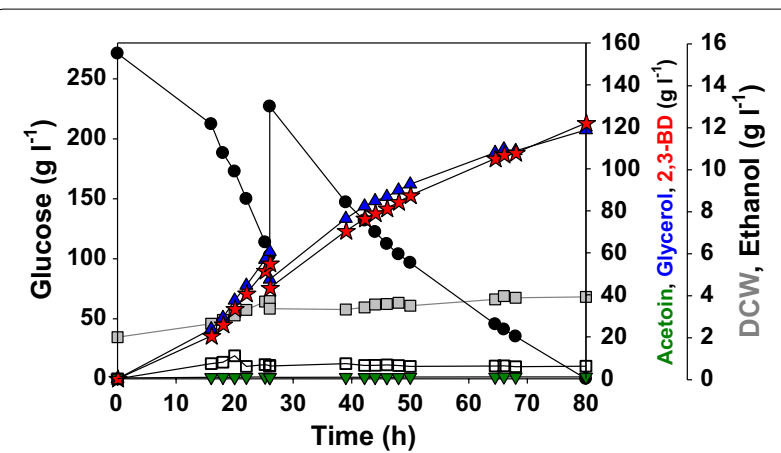

Fig. 2 Fed-batch fermentation profiles of the BD5_G1CtPDC1 strain in YP medium with bioreactor. Symbols: Glucose (filled circle), dry cell weight (gray square), glycerol (blue triangle), acetoin (green inverted triangle), 2,3-BD (red star), and ethanol (open square)

be oxidized in order to reduce glycerol formation and increase 2,3-BD yield. It has been reported that the expression of $L$. lactis NADH oxidase in S. cerevisiae suppressed glycerol formation by reducing intracellular $\mathrm{NADH} / \mathrm{NAD}^{+}$levels [18]. As such, L. lactis NADH oxidase was additionally expressed in the BD5_G1CtPDC1 strain. Because molecular oxygen acts as a substrate for the NADH oxidase reaction, aeration conditions could influence the in vivo activity of NADH oxidase. In order to evaluate the effects of NADH oxidase expression on 2,3-BD production, the batch fermentations with the BD5_G1CtPDC1 and BD5_G1CtPDC1_nox strains were performed under low (25\%), medium (50\%), and high $(100 \%)$ aeration conditions. As expected, the yields of 2,3-BD and glycerol significantly changed according to the levels of aeration (Fig. 3). Under the high aeration

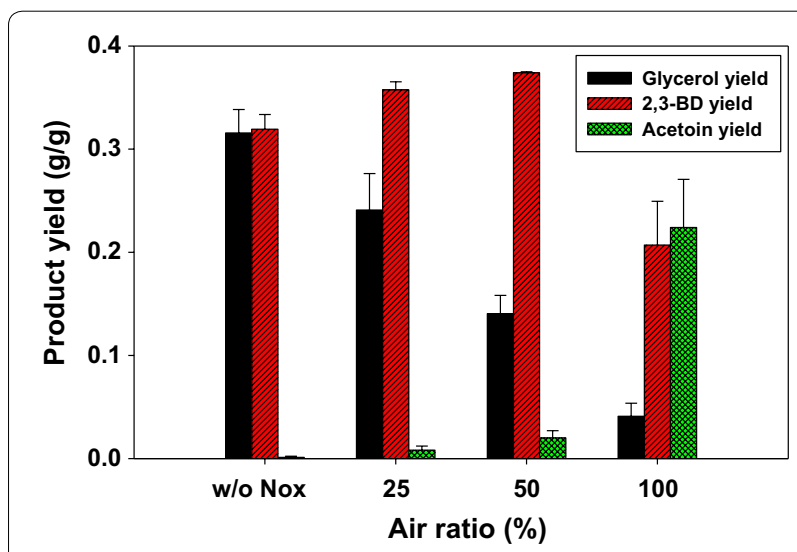

Fig. 3 Glycerol, 2,3-BD, and acetoin yields of the BD5_G1CtPDC1 strain (w/o Nox), and the BD5_G1CtPDC1_nox strain $(25,50,100)$ in batch cultivation with bioreactor. Aeration conditions were controlled by ratios of mixed inlet gas at constant agitation speed of $500 \mathrm{rpm}$ and air flow rate of 2 vvm: only air (w/o Nox and 100), 1:1 mixture of air and nitrogen gas (50), 1:3 mixture of air and nitrogen gas (25) 
condition, the glycerol yield of the BD5_G1CtPDC1_nox strain $(0.041 \pm 0.013 \mathrm{~g}$ glycerol $/ \mathrm{g}$ glucose) was only $12.3 \%$ of that of the BD5_G1CtPDC1 strain $(0.316 \pm 0.022 \mathrm{~g}$ glycerol/g glucose). Interestingly, a large amount of acetoin $(0.224 \pm 0.047 \mathrm{~g}$ acetoin/g glucose) was produced by BD5_G1CtPDC1_nox under the high aeration condition, suggesting that surplus NADH was oxidized by NADH oxidase and generated an NADH-deficient condition. The highest $2,3-\mathrm{BD}$ yield $(0.374 \pm 0.001 \mathrm{~g} 2,3$ - $\mathrm{BD} / \mathrm{g}$ glucose) was observed under the medium aeration condition. The influence of in vivo activity of NADH oxidase on NADH and NAD ${ }^{+}$concentrations in the BD5_ G1CtPDC1_nox strain was analyzed (Additional file 1: Fig. S5). While the NADH/NAD ${ }^{+}$ratio (0.74) under the low aeration condition was similar to that under the medium aeration condition $(0.72)$, the NADH/NAD ${ }^{+}$ratio under the high aeration condition decreased substantially $(0.45)$.

\section{In vivo activity of NADH oxidase influence on intracellular metabolites}

Our results from NADH oxidase expression in the Pdc-deficient strain suggested that cofactor imbalance induced by the deletion of $P D C$ genes in S. cerevisiae could be relieved by the expression of NADH oxidase and control of aeration conditions. To investigate the effects of NADH oxidase at metabolite levels, intracellular metabolites were analyzed from batch fermentations with the BD5_G1CtPDC1 and BD5_G1CtPDC1_nox strains. The batch fermentations were carried out under different conditions including aerobic cultivation of the BD5_G1CtPDC1 strain (Ct100), and microaerobic (N50) and aerobic (N100) cultivation of the BD5_G1CtPDC1_ nox strain (Additional file 1: Fig. S6). Additional expression of NADH oxidase led to reduced glycerol yields and increased acetoin yields regardless of aeration conditions. Moreover, glucose consumption rate $(2.68 \mathrm{~g}$ glucose $/ \mathrm{L} \cdot \mathrm{h})$ of the $\mathrm{Ct} 100$ condition increased to $4.35 \mathrm{~g}$ glucose $/ \mathrm{L} \cdot \mathrm{h}$ for the N50 condition and $3.98 \mathrm{~g}$ glucose/L.h for the N100 condition. Maximum DCWs (2.5 g cell/L) under the Ct100 and N50 conditions were similar, but it increased to $3.9 \mathrm{~g} / \mathrm{L}$ under the $\mathrm{N} 100$ condition.

From the BD5_G1CtPDC1 and BD5_G1CtPDC1_nox cells harvested at $22 \mathrm{~h}, 129$ intracellular metabolites were identified and quantified (Additional file 1: Table S2). These metabolites belonged to different chemical classes including amino acids, organic acids, sugars and sugar alcohols, fatty acids, phosphates, amines, nucleotides. Multivariate data were analyzed by principal component analysis (PCA) (Additional file 1: Fig. S7) and hierarchical clustering analysis (HCA) (Additional file 1: Fig. S8) to explore the variations of intracellular metabolites in response to NADH oxidase expression. As shown in Additional file 1: Fig. S7 and Additional file 1:
Fig. S8, the samples from each condition (Ct100, N50, and N100) were clearly distinguished. It indicated that in vivo activity of NADH oxidase changed intracellular metabolism and displayed distinctive metabolic characteristics. The differences in intracellular metabolites of glycolysis, TCA cycle, and amino acid synthesis between Ct100 and N50 conditions were depicted in Additional file 1: Fig. S9. Intermediate metabolites in the glycolytic pathway including glucose, glucose-6-phosphate, fructose-6-phosphate, and pyruvate, and in the TCA cycle including isocitrate and fumarate significantly increased in Ct100 condition compared to N50 condition.

\section{Fed-batch fermentation with the engineered strain co-expressing CtPDC1 and noxE}

A fed-batch fermentation was carried out to examine whether the BD5_G1CtPDC1_nox strain can be a promising strain to produce 2,3-BD (Fig. 4). Aeration conditions were controlled in two stages to maximize 2,3-BD and minimize by-products formation according to the batch fermentation results shown in Fig. 3. In the first stage, fermentation medium was fully aerated for suppressing glycerol formation. As a result, $97.9 \mathrm{~g} / \mathrm{L}$ of 2,3$\mathrm{BD}$ and $16.7 \mathrm{~g} / \mathrm{L}$ of acetoin were produced at $42.3 \mathrm{~h}$. In the second stage where oxygen-limited conditions were maintained, the produced acetoin was converted into 2,3BD. Finally, $154.3 \mathrm{~g} / \mathrm{L}$ of $2,3-\mathrm{BD}$ was produced with an impressive volumetric productivity of $1.98 \mathrm{~g} 2,3-\mathrm{BD} / \mathrm{L} / \mathrm{h}$. The overall yield of 2,3-BD was $0.404 \mathrm{~g} 2,3 \mathrm{BD} / \mathrm{g}$ glucose which is $80.7 \%$ of the theoretical maximum yield. Glycerol yield ( $0.088 \mathrm{~g}$ glycerol/g glucose) decreased by $73.6 \%$ and final DCW $(6.1 \mathrm{~g} / \mathrm{L})$ increased by a 1.6-fold as compared with those of the BD5_G1CtPDC1 strain in fedbatch fermentation (Fig. 2). Acetate was produced after $30 \mathrm{~h}$, and the final acetate concentration reached $2.3 \mathrm{~g}$ acetate/L.

\section{Discussion}

It has been known that Pdc-deficient S. cerevisiae cannot grow on glucose as a sole carbon source because of $\mathrm{C}_{2}$-auxotrophy and redox imbalance in the cytosol [10]. However, as shown in Additional file 1: Fig. S1B, the engineered strain with the 2,3-BD pathway grew slowly in synthetic medium with glucose as a sole carbon source. The growth recovery of Pdc-deficient $S$. cerevisiae by introduction of the 2,3-BD pathway was also observed in the other studies [32]. In the engineered strains harboring the 2,3-BD biosynthetic genes, $\mathrm{C}_{2}$-compounds might be supplied by $\alpha$-acetolactate synthase (AlsS) which has a decarboxylase activity on pyruvate [33]. In addition, redox imbalance could be relieved by the 2,3-BD biosynthetic pathway because NADH could be additionally oxidized by producing 2,3-BD from pyruvate. However, 

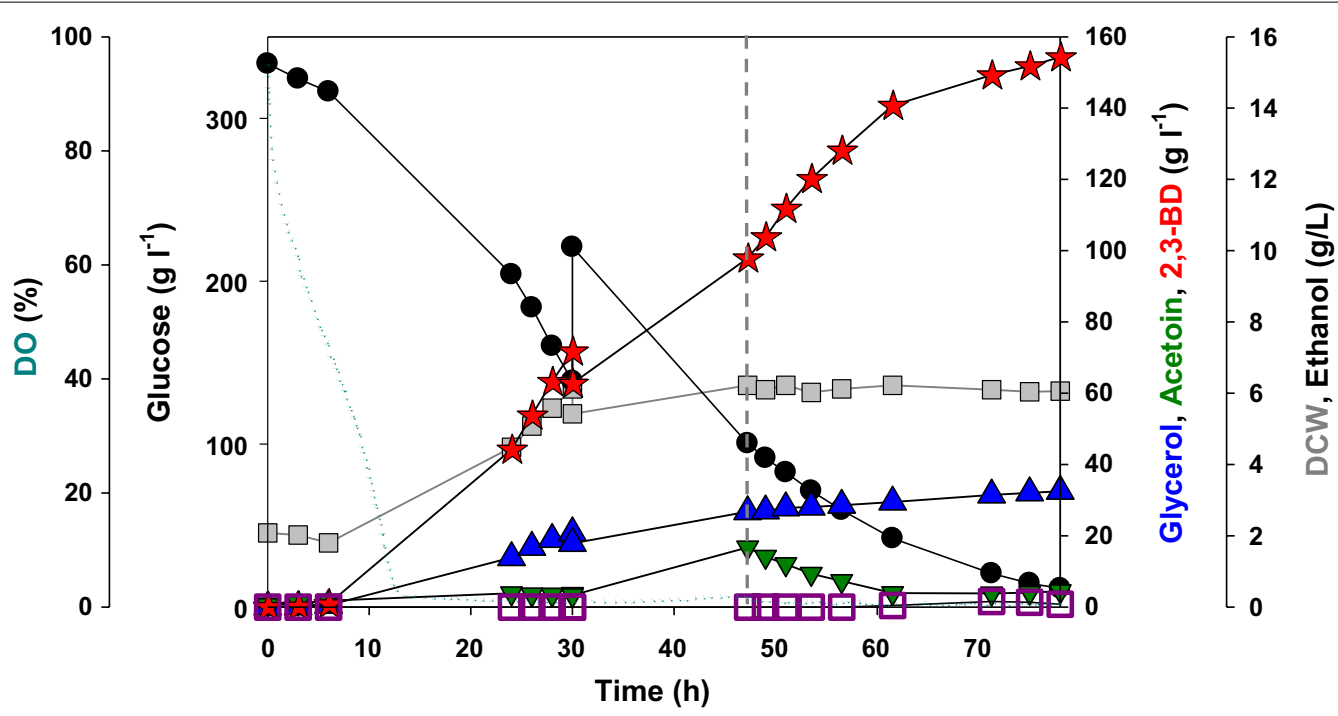

Fig. 4 Fed-batch fermentation profiles of the BD5_G1CtPDC1_nox strain in YP medium with bioreactor. The dashed line represents a shift of aeration condition from $500 \mathrm{rpm} / 2 \mathrm{vvm}$ to $300 \mathrm{rpm} / 1 \mathrm{vvm}$. Symbols: DO (dot), glucose (filled circle), dry cell weight (gray square), glycerol (blue triangle), acetoin (green inverted triangle), 2,3-BD (red star), and ethanol (open square)

the growth recovery by introduction of the 2,3-BD pathway is still insufficient for efficient production of 2,3-BD, and hence additional metabolic engineering is needed for enhancing growth rate, 2,3-BD yield, and productivity.

In other studies, the use of the MTH1 variant has been regarded as an efficient way to overcome the growth defect of Pdc-deficient S. cerevisiae on glucose [12, 21]. The specific growth rate was substantially increased by the mutation on the MTH1 gene (ref, Additional file 1: Fig. S1). However, suppressed expression of hexose transporters by mutation on the $M T H 1$ gene $[34,35]$ became another problem for the efficient production of 2,3-BD. The specific glucose uptake rate of the BD4 strain harboring the MTH1 mutation was only $20 \%$ that of the BD5 strain with the wild-type MTH1 gene (Additional file 1: Fig. S10). As a result, the volumetric productivity of the BD5 strain was much higher than that of the BD4 strain when the growth rate of the BD5 strain was enhanced by supplementation of ethanol as a C2-compound (Additional file 1: Fig. S1C, D). Therefore, we constructed the engineered Pdc-deficient $S$. cerevisiae strain to overcome $\mathrm{C}_{2}$-auxotrophy and redox balance without $M T H 1$ mutation.

As shown in Fig. 1, the product yields and 2,3-BD productivities were changed according to the levels of Pdc activity. The BD5_Con strain produced 2,3-BD dominantly, but ethanol production was positively correlated with Pdc activity. The BD5_G1CtPDC1 strain was selected as the best 2,3-BD production strain because ethanol production was minimized along with the sufficient supplementation of a $\mathrm{C}_{2}$-compound for cell growth.
The production of ethanol by additional $P D C$ expression led to reduced glycerol production because cofactor imbalance might be relieved by ethanol biosynthesis which is a redox-neutral process [36]. As a result, through fine-tuning expression levels of $\mathrm{Pdc}, \mathrm{C}_{2}$-compounds were efficiently supplied for synthesizing acetyl-CoA and 2,3$\mathrm{BD}$ or ethanol production could be easily modulated.

Expression of NADH oxidase in 2,3-BD-producing Pdc-deficient S. cerevisiae reduced glycerol production as a by-product by relieving cofactor balance in the cytosol (Fig. 3). L. lactis $\mathrm{NADH}$ oxidase is an $\mathrm{H}_{2} \mathrm{O}$ forming NADH oxidase involved in the oxygen defense mechanism in facultative anaerobic bacteria [37]. Several studies about the effect of water-forming NADH oxidase in S. cerevisiae strains have been reported [18, 38-40]. Especially, L. lactis NADH oxidase has a lower $\mathrm{Km}$ value $(4.1 \mu \mathrm{M})$ for $\mathrm{NADH}$ [41] than other metabolic enzymes such as Gpd $(23 \mu \mathrm{M})$ and Bdh $(55 \mu \mathrm{M})$ [42, 43], the expression of NADH oxidase caused a large change in metabolic fluxes. The expression of NADH oxidase in S. cerevisiae reduced overflow metabolism [38] and decreased production of reduced metabolites such as ethanol, succinate, 2-hydroxyglutarate, glycerol, and xylitol $[39,40]$. In some cases, the introduction of NADH oxidase led to reduced biomass formation because of decreasing the NADH pool and accumulating acetaldehyde at toxic levels [38, 40]. However, introduction of NADH oxidase efficiently increased 2,3-BD yield and productivity by relieving cofactor imbalance. The possible reasons for this difference are as follows. First, Pdc-deficient $S$. cerevisiae with the 2,3-BD production pathway 
produces excess NADH in the cytosol. Two moles of $\mathrm{NADH}$ were generated by converting one mole of glucose into two moles of pyruvate, but each mole of NADH could be oxidized by producing one mole of 2,3-BD from two moles of pyruvate. The depletion of cytosolic NADH by NADH oxidase might be prevented because the excess cytosolic NADH was generated in the engineered strain. Second, as shown in Fig. 3 and Additional file 1: Fig. S5, the in vivo activity of NADH oxidase could be controlled by aeration condition. As the cells expressing L. lactis $\mathrm{NADH}$ oxidase had a high demand for dissolved oxygen (DO) [40] and rapidly reduced DO levels in fed-batch fermentation [18], the oxygen-limited cultivation could be carried out regardless of aerobic condition. Thus, the optimized aeration condition was able to efficiently enhance 2,3-BD yield and productivity and to reduce byproduct formation (Fig. 4). Third, in fully aerated condition, acetoin which is the oxidized form of 2,3-BD was over-produced by the 2,3-BD-producing Pdc-deficient $S$. cerevisiae strain. Acetoin exerts no deleterious effect in contrast to acetaldehyde, which can inhibit growth and carbon utilization at concentrations above $1.0 \mathrm{~g} / \mathrm{L}$ [44]. In addition, acetoin could be converted into 2,3-BD under oxygen-limited condition (Fig. 4). Thus, acetoin could be acted as a redox sink in the engineered strain for maintaining redox balance that could be induced by excessive NADH oxidase activity.

Interestingly, the expression of NADH oxidase increased glucose uptake rate as well as altered product yields. Similarly, the presence of NADH oxidase or alternative oxidase improved specific glucose uptake rate and subsequent metabolism in the wild-type background [38]. The metabolomic analysis led to a possible hypothesis for this. The concentrations of glucose, and glucose-6-phosphate, and pyruvate (intermediates of glycolysis) increased in the BD5_G1CtPDC1 strain (Ct100) compared with the BD5_G1CtPDC1_nox strain (N50). Pyruvate formation by glycolysis is NADH-generating process. Since cofactor imbalance by reduced oxidation of cytosolic NADH inhibited glycolytic flux and cell growth rate in Pdc-deficient S. cerevisiae [12, 21], the inhibition of glycolytic flux in the BD5_G1CtPDC1 strain might reduce the accumulation of glycolytic intermediates. Metabolic reactions with isocitrate and fumarate as substrate are also NADH-generating steps in the TCA cycle. Similar to glycolytic intermediates, these compounds might accumulate because of intracellular cofactor imbalance of NADH in the BD5_G1CtPDC1 strain (Ct100). From the comparison of intracellular metabolites in the BD5_G1CtPDC1 and BD5_G1CtPDC1_nox strains, the relieved cofactor imbalance by $\mathrm{NADH}$ oxidase changed glycolytic and TCA cycle intermediates. These data could explain the improved glucose consumption rate by the expression of $\mathrm{NADH}$ oxidase in Pdc-deficient S. cerevisiae.

\section{Conclusions}

In this study, a supplementation of a $\mathrm{C}_{2}$-compound by optimized expression of Pdc and relieved cofactor imbalance by controlled in vivo activity of $\mathrm{NADH}$ oxidase enhanced 2,3-BD yield, productivity, and titer in 2,3-BDproducing Pdc-deficient $S$. cerevisiae. The 2,3-BD productivity was increased fivefold compared to the previous report [12]. In addition, final titer of 2,3-BD (154.3 g/L) is one of the highest concentration reported for production of 2,3-BD by engineered yeasts and bacterial native producers. To improve 2,3-BD production performance by the engineered yeast strains, efficient utilization of various sugars such as galactose, xylose, and cellobiose or elimination of glycerol formation are required. In addition, optimizing aeration condition is important in largescale fermentations. The fine-tuned expression of $P D C$ and co-expression of NADH oxidase in Pdc-deficient $S$. cerevisiae could be applied to improving other chemical production systems using pyruvate as a precursor compound.

\section{Methods}

\section{Construction of plasmids}

Strains and plasmids used in this study are summarized in Table 1. The primers used for cloning of the $P D C$ genes from various yeast species and AUR1-C gene are listed in Table 2. E. coli TOP10 (Invitrogen, Carlsbad, CA) was used for gene cloning and manipulation. E. coli transformants were grown in Lysogeny Broth (LB) medium with $100 \mu \mathrm{g} / \mathrm{mL}$ of ampicillin. To construct expression plasmids with different $P D C$ genes, ORFs of $P D C$ genes were amplified by PCR from genomic DNA of $C$. tropicalis, Kluyveromyces marxianus, and S. cerevisiae using the primers in Table 2. The amplified DNA fragments were ligated into appropriate restriction sites in $\mathrm{p} 426 \mathrm{GPD}$, p406CYC1, and p406GPD2 plasmids. In order to construct aureobasidin A resistance plasmids, the AUR1-C gene was amplified from pAUR101 plasmids by PCR with primers of F_SnaBI_AUR1-C and R_MfeI_AUR1-C. The amplified DNA fragments were ligated into the corresponding restriction sites in Table 2.

\section{Yeast transformation and construction of recombinant $S$. cerevisiae strains}

Transformation of plasmids for constructing engineered strains was performed using a spheroplast transformation kit (BIO 101, Vista, CA). To select transformants, S. cerevisiae strains were routinely cultivated aerobically at $30{ }^{\circ} \mathrm{C}$ in $\mathrm{YNB}$ medium $(6.7 \mathrm{~g} / \mathrm{L}$ yeast nitrogen base and appropriate nucleotides and amino acid). $20 \mathrm{~g} / \mathrm{L}$ 
Table 2 Primers used in this study

\begin{tabular}{|c|c|c|}
\hline Primers & Restriction site & Sequence \\
\hline \multicolumn{3}{|c|}{ Cloning of PDC genes } \\
\hline F_Xmal_CtPDC1 & Xmal & tcccCCCGGGaaaatgtctgaaattactttgggtag \\
\hline R_Sall_CtPDC1 & Sall & acgcGTCGACtttattcttgagcagcgttg \\
\hline F_Xmal_KmPDC1 & Xmal & tcccCCCGGGaaaatgtctgaaattactctaggtcg \\
\hline R_Sall_KmPDC1 & Sall & acgcGTCGACtttattcttgcttggcgtt \\
\hline F_Xmal_ScPDC1 & Xmal & tcccCCCGGGatgtctgaaattactttgggtaa \\
\hline R_Sall_ScPDC1 & Sall & acgcGTCGACttattgcttagcgttggtag \\
\hline F_Xmal_ScPDC5 & Xmal & tcccCCCGGGatgtctgaaataaccttaggtaaata \\
\hline R_Sall_ScPDC5 & Sall & acgcGTCGACttattgtttagcgttagtagcg \\
\hline F_Xmal_ScPDC6 & Xmal & tcccCCCGGGatgtctgaaattactcttggaaaatac \\
\hline R_Sall_ScPDC6 & Sall & acgcGTCGACttattgtttggcatttgtagc \\
\hline \multicolumn{3}{|c|}{ Cloning of AUR1-C gene } \\
\hline F_SnaBI_AUR1-C & SnaBI & agcttgtcacctTACGTAaaagtgcccatcagtgttc \\
\hline R_Mfel_AUR1-C & Mfel & ataaccgggtCAATTG cagaggaaagaataacgcaa \\
\hline
\end{tabular}

Bold and capital characters are restriction enzyme sites

of ethanol was used as a carbon source for the cultivation of the SOS5 strain and $20 \mathrm{~g} / \mathrm{L}$ of glucose and $1 \mathrm{~g} / \mathrm{L}$ of ethanol was used for cultivation of the other strains. The $C$. tropicalis $P D C$ gene $(C t P D C 1), K$. marxianus $P D C$ gene (KmPDC1), and S. cerevisiae PDC genes (ScPDC1, $S c P D C 5, S c P D C 6)$ were expressed under TDH3 promoter and multi-copy plasmid into the SOS5 strain. The $C$. tropicalis $P D C$ gene was expressed under different native promoters (TDH3, CYC1, and GPD2) and copy number (single and multi-copy). The $P D C$ expression plasmids in Table 1 were introduced into the SOS5 and BD5 strains and the engineered $S$. cerevisiae strains expressing different levels of $P D C$ were constructed. Integrative $P D C$ expression plasmids (p406CYC1_CtPDC1, p406GPD2 CtPDC1, and p406TDH3_CtPDC1) were digested with $S t u \mathrm{I}$ before use and integrated into the URA3 locus. For additional expression of $L$. lactis NADH oxidase, pAUR Llnox plasmid was transformed to the BD5_G1CtPDC1 strain. The resulting recombinant $S$. cerevisiae strains are listed in Table 1.

\section{Fermentation conditions}

All cultures were carried out at $30{ }^{\circ} \mathrm{C}$. Pre-cultures of yeast cells were conducted aerobically in $250-\mathrm{mL}$ baffled flasks. Main flask batch cultures were conducted under microaerobic conditions in $250-\mathrm{mL}$ flasks at $80 \mathrm{rpm}$. In order to prepare inoculums, engineered $S$. cerevisiae cells were cultivated for $48-72 \mathrm{~h}$ in $5 \mathrm{~mL}$ YNB medium containing $20 \mathrm{~g} / \mathrm{L}$ glucose and $1 \mathrm{~g} / \mathrm{L}$ ethanol. The grown cells were transferred to $100 \mathrm{~mL}$ YNB medium containing $20 \mathrm{~g} / \mathrm{L}$ glucose and $0.5 \mathrm{~g} / \mathrm{L}$ ethanol. After $24 \mathrm{~h}$ cultivation, the mid-exponential growing cells $\left(\mathrm{OD}_{600}<3\right)$ were harvested and washed twice with double-distilled water (DDW). Cells were inoculated into the main culture at the initial concentration of $0.20 \mathrm{~g} / \mathrm{L}$. The main culture YP medium $(10 \mathrm{~g} / \mathrm{L}$ yeast extract and $20 \mathrm{~g} / \mathrm{L}$ peptone) contains $100 \mathrm{~g} / \mathrm{L}$ glucose, and YNB medium contains $90 \mathrm{~g} / \mathrm{L}$ glucose and $50 \mathrm{mM}$ potassium phthalate at pH 5.5 adjusted by $\mathrm{NaOH}$. Ethanol $0.5 \mathrm{~g} / \mathrm{L}$ was added if necessary. Batch fermentations with bioreactor were performed in $500 \mathrm{~mL}$ YP medium containing $100 \mathrm{~g} / \mathrm{L}$ glucose using $1 \mathrm{~L}$ bench-top fermentor (Fermentec, Korea). For controlling aeration conditions, different ratios of mixed inlet gas (pure air, 1:1 or 1:3 of air and nitrogen gas mixture) were used at constant agitation speed of $500 \mathrm{rpm}$ and air flow rate of $2 \mathrm{vvm}$. Fed-batch fermentations with bioreactor were carried out in $500 \mathrm{~mL}$ YP medium containing $300 \mathrm{~g} / \mathrm{L}$ glucose using $1 \mathrm{~L}$ bench-top fermentor (Fermentec, Korea) at $30^{\circ} \mathrm{C}$. The medium pH was maintained at 5.5 with $5 \mathrm{~N} \mathrm{NaOH}$ solution, and dissolved oxygen (DO) levels were monitored with $\mathrm{O}_{2}$ sensor (Mettler Toledo, Switzerland). The culture medium was agitated at $300-500 \mathrm{rpm}$ and aerated with air flow rate of $1-2 \mathrm{vvm}$ according to DO levels in medium. During the fed-batch fermentation, DO levels were kept under 2.0. The grown cells prepared from flask culture were inoculated at the initial concentration of $2.0 \mathrm{~g} / \mathrm{L}$.

\section{Analysis of dry cell weight and metabolites}

Cell growth was monitored by optical density at $600 \mathrm{~nm}$ $\left(\mathrm{OD}_{600}\right)$ using a spectrophotometer (UV-1601, Shimadzu, Japan). Dry cell weight (DCW) was calculated using a predetermined factor of $0.20 \mathrm{~g}$ DCW/L/OD ${ }_{600}$ [36]. Glucose, glycerol, acetoin, 2,3-BD, and ethanol were analyzed by a high-performance liquid chromatography (1100 series, Agilent, CO) equipped with a Rezex ROA-organic acid 
column (Phenomenex, CA). Metabolites were detected by a refractive index (RI) detector. For measurement of intracellular NADH and NAD ${ }^{+}$, about $4 \times 10^{7}$ of exponentially growing cells in flask batch cultivation were used. NADH and $\mathrm{NAD}^{+}$concentration assays were conducted following manufacturer-provided methods (BioAssay Systems, CA).

\section{In vitro Pdc activity analysis}

To prepare crude extracts, about $1 \times 10^{9}$ mid-exponential phase cells grown on the YNB medium with $80 \mathrm{~g} / \mathrm{L}$ glucose and $0.5 \mathrm{~g} / \mathrm{L}$ ethanol in a flask culture were harvested and washed twice with DDW. Protease inhibitor (Roche, Switzerland) was added, and the harvested cells were lysed with Yeast Protein Extraction Reagent (Y-PER, Thermo Scientific, MA). After centrifugation for $20 \mathrm{~min}$ at $12,000 \mathrm{rpm}$ and $4{ }^{\circ} \mathrm{C}$, the supernatants were used to determine the Pdc activity within $3 \mathrm{~h}$ and diluted with DDW if necessary. The NADH oxidase activity assays were performed at $30{ }^{\circ} \mathrm{C}$ with the reaction mixture containing $40 \mathrm{mM}$ imidazole hydrochloride buffer ( $\mathrm{pH} 6.5)$, $5 \mathrm{mM} \mathrm{MgCl} 2,0.2 \mathrm{mM}$ TPP, $10 \mathrm{U}$ alcohol dehydrogenase from $S$. cerevisiae, $0.4 \mathrm{mM} \mathrm{NADH}$, and $50 \mathrm{mM}$ pyruvate [27]. The reactions were initiated by adding pyruvate, and a decrease of absorbance at $340 \mathrm{~nm}$ was measured. One unit of activity was defined as the amount of enzyme oxidizing $1 \mu \mathrm{mol} \mathrm{NADH}$ per minute at the corresponding reaction conditions. The protein concentration of crude extracts was determined by the Bradford method [45]. For determination of kinetic values $\left(K_{\mathrm{m}}\right.$ and $\left.V_{\max }\right)$ of the Pdc enzymes, Pdc activities were measured at various concentrations of pyruvate $(1.56,3.13,6.25,12.5$, 25.0, 50.0, $100 \mathrm{mM}$ ). The $K_{\mathrm{m}}$ and $V_{\max }$ values for Pdc were determined from Lineweaver-Burk plots of the data.

\section{Sample preparation for metabolite analysis}

For the extraction of metabolome sampling, a fast filtration method was performed with a slight modification of the method used in a previous study [46]. Briefly, cell pellets on each conditions were collected by vacuumfiltering of $1-\mathrm{mL}$ culture broth using a nylon membrane filter $(0.45 \mu \mathrm{m}$ pore size, $30 \mathrm{~mm}$ diameter; Whatman, Piscataway, NJ) and washed using $5 \mathrm{~mL}$ of distilled water at room temperature. The membrane filter and loaded cells were mixed using $10 \mathrm{~mL}$ of acetonitrile/water $(1: 1, \mathrm{v} / \mathrm{v})$ at $-20^{\circ} \mathrm{C}$. After that, the extraction mixture was immersed in liquid nitrogen. These steps were completed in less than $30 \mathrm{~s}$. The extraction mixture was thawed on ice, vortexed for $3 \mathrm{~min}$, and centrifuged at 16,100 $\times g$ for $5 \mathrm{~min}$ at $4{ }^{\circ} \mathrm{C}$. The supernatant was collected and vacuum-dried.

\section{GC/TOF MS analysis}

For the GC/TOF MS analysis, the derivative metabolite samples were prepared using $5 \mu \mathrm{L}$ of $40 \mathrm{mg} / \mathrm{mL}$ methoxyamine hydrochloride in pyridine (Sigma-Aldrich, St. Louis, MO) at $30^{\circ} \mathrm{C}$ for $90 \mathrm{~min}$ and $45 \mu \mathrm{L}$ of $\mathrm{N}$-methyl$N$-trimethylsilyl trifluoroacetamide (Fluka, Buchs, Switzerland) at $37^{\circ} \mathrm{C}$ for $30 \mathrm{~min}$. As retention index markers, a mixture of fatty acid methyl esters was added to the derivative samples. An Agilent 7890A FX (Agilent Technologies, Wilmington, DE) coupled with a Pegasus HT TOF MS (LECO, St. Joseph, MI) was used for metabolite analysis. A $0.5 \mu \mathrm{L}$ of derivative samples was injected into the GC in splitless mode and separated on an RTX-5Sil MS column $(30 \mathrm{~m} \times 0.25 \mathrm{~mm}$ i.d., $0.25 \mu \mathrm{m}$ film thickness, Restek, Bellefonte, PA) with an additional 10-m integrated guard column. The initial oven temperature was set at $50{ }^{\circ} \mathrm{C}$ for $1 \mathrm{~min}$ and then increased to $330^{\circ} \mathrm{C}$ at a rate of $20^{\circ} \mathrm{C} / \mathrm{min}$ and held at $330^{\circ} \mathrm{C}$ for $5 \mathrm{~min}$. Mass spectra were recorded in the mass range of $85-500 \mathrm{~m} / z$ at an acquisition rate of ten spectra/s. The temperatures of the ion source and transfer line were set at 250 and $280^{\circ} \mathrm{C}$, respectively, and the sample ionization was performed electron impact at $70 \mathrm{eV}$.

\section{Data processing and statistical analysis for metabolomics analysis}

For the processing of GC/TOF MS data, the software LECO Chroma TOF was used to detect peaks and to deconvolute the mass spectra. The processed data were further processed using an inhouse library, BinBase [47]. The raw metabolite data were normalized by the median of the sum of the peak intensities of all identified metabolites in each sample. The normalized data were used for multivariate and univariate statistical analysis [48], and MultiExperiment Viewer was used for the hierarchical clustering analysis (HCA).

\section{Additional file}

Additional file 1: Table S1. Kinetic constants $\left(K_{m}\right.$ and $\left.V_{\max }\right)$ of $P d c$ enzymes, Figure $\mathbf{S 1}$. Batch cultivation of the BD4 strain and the BD5 strain in minimal medium, Figure S2. Amino acid sequence of the $C$. tropicalis pyruvate decarboxylase I, Figure S3. In vitro Pdc activities in the control and four engineered S. cerevisiae strains expressing C. tropicalis pyruvate decarboxylase gene (CtPDC1) differentially, Figure S4. Fermentation profiles of the CtPDC1 expressing strains with glucose as a sole carbon source in minimal medium, Figure S5. The NADH and $\mathrm{NAD}^{+}$concentrations in the BD5 G1CtPDC1_nox strain with various aeration conditions, Figure S6. Profiles of batch cultivations for metabolomic analysis, Figure S7. Principal component analysis (PCA) of intracellular metabolite profiles of BD5_G1CtPDC1 and BD5_G1CtPDC1_nox in different aerobic conditions, Figure S8. Hierarchical clustering analysis (HCA) of metabolite profiles of BD5_G1CtPDC1 and BD5_G1CtPDC1_nox in different aerobic conditions, Figure S9. Comparison of intracellular metabolites between BD5_G1CtPDC1 at aerobic condition (Ct100) and BD5_G1CtPDC1_nox at microaerobic condition (N50), Figure S10. Specific glucose uptake rates of the wild type D452-2 and engineered strains.

\section{Authors' contributions}

JWK conceived and designed the study, analyzed and interpreted data, conducted experiments, and wrote the manuscript. JK and KHK operated GC/ TOF MS and did the analysis of metabolomics study part. SOS contributed 
to design and construct recombinant strains. YSJ and JHS analyzed data and revised the manuscript. All authors read and approved the final manuscript.

\section{Author details}

1 Department of Agricultural Biotechnology, Center for Food and Bioconvergence, Seoul National University, Seoul 151-921, Republic of Korea. ${ }^{2}$ School of Life Sciences and Biotechnology, Korea University, Seoul 136-713, Republic of Korea. ${ }^{3}$ Department of Food Science and Human Nutrition, Institute for Genomic Biology, University of Illinois at Urbana-Champaign, Urbana, IL 61801, USA

\section{Acknowledgements}

This work was supported by the Advanced Biomass R\&D Center (ABC) of Global Frontier Project (2011-0031359) and the National Research Foundation of Korea Grant (2014M1 A2A2069904) funded by the Ministry of Science, ICT, and Future Planning.

\section{Competing interests}

The authors declare that they have no competing interests.

\section{Availability of data and materials}

Data made available to all interested researchers upon request.

\section{Consent for publication}

All authors approved the manuscript.

\section{Funding}

This study is funded by National Research Foundation of Korea.

Received: 19 September 2016 Accepted: 1 December 2016 Published online: 09 December 2016

\section{References}

1. Syu M-J. Biological production of 2, 3-butanediol. Appl Microbiol Biotechnol. 2001;55(1):10-8.

2. Ryu C-M, Farag MA, Hu C-H, Reddy MS, Wei H-X, Paré PW, Kloepper JW Bacterial volatiles promote growth in Arabidopsis. Proc Natl Acad Sci USA. 2003;100(8):4927-32.

3. Ji X-J, Huang H, Ouyang P-K. Microbial 2, 3-butanediol production: a state-of-the-art review. Biotechnol Adv. 2011;29(3):351-64.

4. Cho S, Kim T, Woo HM, Kim Y, Lee J, Um Y. High production of 2, 3-butanediol from biodiesel-derived crude glycerol by metabolically engineered Klebsiella oxytoca M1. Biotechnol Biofuels. 2015;8(1):1.

5. Häßler T, Schieder D, Pfaller R, Faulstich M, Sieber V. Enhanced fed-batch fermentation of 2, 3-butanediol by Paenibacillus polymyxa DSM 365. Bioresour Technol. 2012:124:237-44.

6. Jantama K, Polyiam P, Khunnonkwao P, Chan S, Sangproo M, Khor K, Jantama SS, Kanchanatawee S. Efficient reduction of the formation of by-products and improvement of production yield of 2, 3-butanediol by a combined deletion of alcohol dehydrogenase, acetate kinasephosphotransacetylase, and lactate dehydrogenase genes in metabolically engineered Klebsiella oxytoca in mineral salts medium. Metab Eng. 2015:30:16-26.

7. Celińska E, Grajek W. Biotechnological production of 2, 3-butanediolcurrent state and prospects. Biotechnol Adv. 2009;27(6):715-25.

8. Pronk JT, Steensma HY, Van Dijken JP. Pyruvate metabolism in Saccharomyces cerevisiae. Yeast. 1996;12(16):1607-33.

9. Flikweert MT, van der Zanden L, Janssen WMTM, Yde Steensma H, van Dijken JP, Pronk JT. Pyruvate decarboxylase: an indispensable enzyme for growth of Saccharomyces cerevisiae on glucose. Yeast. 1996;12(3):247-57.

10. Flikweert MT, Swaaf M, Dijken JP, Pronk JT. Growth requirements of pyruvate-decarboxylase-negative Saccharomyces cerevisiae. FEMS Microbiol Lett. 1999;174(1):73-9.

11. van Maris AJ, Geertman JMA, Vermeulen A, Groothuizen MK, Winkler AA, Piper MD, van Dijken JP, Pronk JT. Directed evolution of pyruvate decarboxylase-negative Saccharomyces cerevisiae, yielding a $C_{2}$-independent, glucose-tolerant, and pyruvate-hyperproducing yeast. Appl Environ Microbiol. 2004;70(1):159-66.
12. Kim S-J, Seo S-O, Jin Y-S, Seo J-H. Production of 2, 3-butanediol by engineered Saccharomyces cerevisiae. Bioresour Technol. 2013;146:274-81.

13. Van Roermund C, Elgersma Y, Singh N, Wanders R, Tabak H. The membrane of peroxisomes in Saccharomyces cerevisiae is impermeable to NAD $(H)$ and acetyl-CoA under in vivo conditions. EMBO J. 1995;14(14):3480.

14. Giaever G, Chu AM, Ni L, Connelly C, Riles L, Veronneau S, Dow S, LucauDanila A, Anderson K, Andre B. Functional profiling of the Saccharomyces cerevisiae genome. Nature. 2002;418(6896):387-91.

15. Bruinenberg PM, Jonker R, van Dijken JP, Scheffers WA. Utilization of formate as an additional energy source by glucose-limited chemostat cultures of Candida utilis CBS 621 and Saccharomyces cerevisiae CBS 8066. Arch Microbiol. 1985;142(3):302-6.

16. Lagunas R, Gancedo JM. Reduced pyridine-nucleotides balance in glucose-growing Saccharomyces cerevisiae. Eur J Biochem. 1973;37(1):90-4.

17. Ng CY, Jung M-y, Lee J, Oh M-K. Production of 2, 3-butanediol in Saccharomyces cerevisiae by in silico aided metabolic engineering. Microb Cell Fact. 2012;11:68.

18. Kim J-W, Seo S-O, Zhang G-C, Jin Y-S, Seo J-H. Expression of Lactococcus lactis NADH oxidase increases 2, 3-butanediol production in Pdc-deficient Saccharomyces cerevisiae. Bioresour Technol. 2015;191:512-9.

19. Lafuente MJ, Gancedo C, Jauniaux JC, Gancedo JM. Mth1 receives the signal given by the glucose sensors Snf3 and Rgt2 in Saccharomyces cerevisiae. Mol Microbiol. 2000;35(1):161-72.

20. Moriya $\mathrm{H}$, Johnston M. Glucose sensing and signaling in Saccharomyces cerevisiae through the Rgt2 glucose sensor and casein kinase I. Proc Natl Acad Sci USA. 2004;101(6):1572-7.

21. Oud B, Flores C-L, Gancedo C, Zhang X, Trueheart J, Daran J-M, Pronk JT, van Maris A. An internal deletion in MTH1 enables growth on glucose of pyruvate-decarboxylase negative, non-fermentative Saccharomyces cerevisiae. Microb Cell Fact. 2012;11:131.

22. Wang A, Xu Y, Ma C, Gao C, Li L, Wang Y, Tao F, Xu P. Efficient 2, 3-butanediol production from cassava powder by a crop-biomass-utilizer, Enterobacter cloacae subsp. dissolvens SDM. PLoS ONE. 2012;7(7):e40442.

23. Petrov K, Petrova P. High production of 2, 3-butanediol from glycerol by Klebsiella pneumoniae G31. Appl Microb Biotech. 2009;84(4):659-65.

24. Kim J-H. DNA-binding properties of the yeast Rgt1 repressor. Biochimie. 2009;91(2):300-3.

25. Fletcher E, Krivoruchko A, Nielsen J. Industrial systems biology and its impact on synthetic biology of yeast cell factories. Biotechnol Bioeng. 2016;113(6):1164-70

26. van Urk H, Voll WL, Scheffers WA, Van Dijken JP. Transient-state analysis of metabolic fluxes in Crabtree-positive and Crabtree-negative yeasts. Appl Environ Microbiol. 1990;56(1):281-7.

27. Postma E, Verduyn C, Scheffers WA, Van Dijken JP. Enzymic analysis of the crabtree effect in glucose-limited chemostat cultures of Saccharomyces cerevisiae. Appl Environ Microbiol. 1989;55(2):468-77.

28. De Deken R. The Crabtree effect: a regulatory system in yeast. J Gen Microbiol. 1966;44(2):149-56.

29. Arjunan P, Umland T, Dyda F, Swaminathan S, Furey W, Sax M, Farrenkopf $B$, Gao Y, Zhang D, Jordan F. Crystal structure of the thiamin diphosphatedependent enzyme pyruvate decarboxylase from the yeast Saccharomyces cerevisiae at 2.3Å resolution. J Mol Biol. 1996;256(3):590-600.

30. Pohl M. Protein design on pyruvate decarboxylase (PDC) by site-directed mutagenesis. In: Scheper T, editor. New enzymes for organic synthesis. Heidelberg: Springer; 1997.

31. Baburina I, Gao Y, Hu Z, Jordan F, Hohmann S, Furey W. Substrate activation of brewers'yeast pyruvate decarboxylase is abolished by mutation of cysteine 221 to serine. Biochemistry. 1994;33(18):5630-5.

32. Nan H, Seo S-O, Oh EJ, Seo J-H, Cate JH, Jin Y-S. 2, 3-Butanediol production from cellobiose by engineered Saccharomyces cerevisiae. Appl Microbiol Biotechnol. 2014;98:5757-64.

33. Atsumi S, Li Z, Liao JC. Acetolactate synthase from Bacillus subtilis serves as a 2-ketoisovalerate decarboxylase for isobutanol biosynthesis in Escherichia coli. Appl Environ Microbiol. 2009;75(19):6306-11.

34. Flick KM, Spielewoy N, Kalashnikova TI, Guaderrama M, Zhu Q, Chang H-C, Wittenberg C. Grr1-dependent inactivation of Mth1 mediates glucoseinduced dissociation of Rgt1 from HXT gene promoters. Mol Biol Cell. 2003;14(8):3230-41.

35. Kim J-H, Brachet $\mathrm{V}$, Moriya $\mathrm{H}$, Johnston $\mathrm{M}$. Integration of transcriptional and posttranslational regulation in a glucose signal transduction pathway in Saccharomyces cerevisiae. Eukaryot Cell. 2006;5(1):167-73. 
36. Kim J-W, Chin Y-W, Park Y-C, Seo J-H. Effects of deletion of glycerol3-phosphate dehydrogenase and glutamate dehydrogenase genes on glycerol and ethanol metabolism in recombinant Saccharomyces cerevisiae. Bioproc Biosyst Eng. 2012;35(1):49-54.

37. Jiang R, Riebel BR, Bommarius AS. Comparison of alkyl hydroperoxide reductase (AhpR) and water-forming $\mathrm{NADH}$ oxidase from Lactococcus lactis ATCC 19435. Adv Synth Catal. 2005;347(7-8):1139-46.

38. Vemuri G, Eiteman M, McEwen J, Olsson L, Nielsen J. Increasing NADH oxidation reduces overflow metabolism in Saccharomyces cerevisiae. Proc Natl Acad Sci USA. 2007;104(7):2402-7.

39. Zhang G-C, Liu J-J, Ding W-T. Decreased xylitol formation during xylose fermentation in Saccharomyces cerevisiae due to overexpression of waterforming NADH oxidase. Appl Environ Microbiol. 2012;78(4):1081-6.

40. Heux S, Cachon R, Dequin S. Cofactor engineering in Saccharomyces cerevisiae: expression of $\mathrm{a}_{2} \mathrm{O}$-forming $\mathrm{NADH}$ oxidase and impact on redox metabolism. Metab Eng. 2006;8(4):303-14.

41. LopezdeFelipe F, Hugenholtz J. Purification and characterisation of the water forming NADH-oxidase from Lactococcus lactis. Int Dairy J. 2001;11(1):37-44.

42. González E, Fernández MR, Larroy C, Solà LS, Pericàs MA, Parés X, Biosca JA. Characterization of a (2R, 3R)-2, 3-butanediol dehydrogenase as the Saccharomyces cerevisiae YALO60W gene product. J Biol Chem. 2000;275(46):35876-85.

43. Albertyn J, van Tonder A, Prior BA. Purification and characterization of glycerol-3-phosphate dehydrogenase of Saccharomyces cerevisiae. FEBS Lett. 1992;308(2):130-2.
44. Roustan JL, Sablayrolles J-M. Modification of the acetaldehyde concentration during alcoholic fermentation and effects on fermentation kinetics. J Biosci Bioeng. 2002;93(4):367-75.

45. Bradford MM. A rapid and sensitive method for the quantitation of microgram quantities of protein utilizing the principle of protein-dye binding. Anal Biochem. 1976;72(1):248-54.

46. Kim S, Lee DY, Wohlgemuth G, Park HS, Fiehn O, Kim KH. Evaluation and optimization of metabolome sample preparation methods for Saccharomyces cerevisiae. Anal Chem. 2013;85(4):2169-76.

47. Lee DY, Fiehn O. High quality metabolomic data for Chlamydomonas reinhardtii. Plant Methods. 2008:4(1):7.

48. Denkert C, Budczies J, Weichert W, Wohlgemuth G, Scholz M, Kind T, Niesporek S, Noske A, Buckendahl A, Dietel M. Metabolite profiling of human colon carcinoma-deregulation of TCA cycle and amino acid turnover. Mol Cancer. 2008;7(1):72.

49. Nikawa J-I, Tsukagoshi Y, Yamashita S. Isolation and characterization of two distinct myo-inositol transporter genes of Saccharomyces cerevisiae. $J$ Biol Chem. 1991;266(17):11184-91.

50. Mumberg D, Müller R, Funk M. Yeast vectors for the controlled expression of heterologous proteins in different genetic backgrounds. Gene. 1995;156(1):119-22

51. Kim S-J, Seo S-O, Park Y-C, Jin Y-S, Seo J-H. Production of 2, 3-butanediol from xylose by engineered Saccharomyces cerevisiae. J Biotechnol. 2014;192:374-82.

\section{Submit your next manuscript to BioMed Central and we will help you at every step:}

- We accept pre-submission inquiries

- Our selector tool helps you to find the most relevant journal

- We provide round the clock customer support

- Convenient online submission

- Thorough peer review

- Inclusion in PubMed and all major indexing services

- Maximum visibility for your research

Submit your manuscript at www.biomedcentral.com/submit 